\title{
Constraining proposed combinations of ice history and Earth rheology using VLBI determined baseline length rates in North America
}

\section{Citation}

Mitrovica, J. X., J. L. Davis, and I. I. Shapiro. 1993. "Constraining Proposed Combinations of Ice History and Earth Rheology Using VLBI Determined Baseline Length Rates in North America." Geophysical Research Letters 20 (21): 2387-90. https://doi.org/10.1029/93gl02043.

\section{Permanent link}

http://nrs.harvard.edu/urn-3:HUL.InstRepos:41401416

\section{Terms of Use}

This article was downloaded from Harvard University's DASH repository, and is made available under the terms and conditions applicable to Other Posted Material, as set forth at http:// nrs.harvard.edu/urn-3:HUL.InstRepos:dash.current.terms-of-use\#LAA

\section{Share Your Story}

The Harvard community has made this article openly available.

Please share how this access benefits you. Submit a story.

Accessibility 


\section{CONSTRAINING PROPOSED COMBINATIONS OF ICE HISTORY AND EARTH RHEOLOGY USING VLBI DETERMINED BASELINE LENGTH RATES IN NORTH AMERICA}

\section{J. X. Mitrovica ${ }^{1}$, J. L. Davis ${ }^{2}$, and I. I. Shapiro ${ }^{2}$}

Abstract. We predict the present-day rates of change of the lengths of 19 North American baselines due to the glacial isostatic adjustment process. Contrary to previously published research, we find that the threedimensional motion of each of the sites defining a baseline, rather than only the radial motions of these sites, needs to be considered to obtain an accurate estimate of the rate of change of the baseline length. Predictions are generated using a suite of Earth models and late Pleistocene ice histories; these include specific combinations of the two which have been proposed in the literature as satisfying a variety of rebound related geophysical observations from the North American region. A number of these published models are shown to predict rates which differ significantly from the VLBI observations.

\section{Introduction}

Glacial isostatic disequilibrium is manifested in a number of geophysical observables, none more apparent than the associated three-dimensional crustal deformations. Radial deformations have received the most attention [e.g. Cathles, 1975]. Horizontal motions, in contrast, have been considered in only a very few studies [e.g., James and Morgan, 1990; Mitrovica et al., 1993a], since there has, until very recently, been an absence of observational constraints on such motions. The continuing inprovement of space-geodetic techniques has enabled three-dimensional crustal deformation rates to be estiinated with an accuracy useful for application to the glacial isostatic adjustment problem. Indeed, Mitrovica et al. [1993a] compared theoretical predictions of three-dimensional motions associated with the adjustment process with available very-long-baseline interferometry (VLBI) constraints from North America and Europe. In particular, Mitrovica et al. [1993a] used the results of Ryan et al. [1993] who analyzed an ensemble of Mark III geodetic VLBI data obtained from 1979-1991 to determine the evolutions of a large number of baselines. The "global" solution of Ryan et al. [1993], which they termed GLB868, included 864,359 group delay measurements obtained in 1648 observing sessions at a globally distributed network of sites.

The typical application of observables related to glacial isostatic adjustment is to constrain the radial profile of mantle viscosity. A complication in most such applications is the sensitivity of the geophysical data to the space and time geometry of the late Pleistocene ice sheets. Mitrovica et al. [1993a] concluded that the VLEI constraints are most usefully applied to assess the acceptability of specific ice model/Earth model pairings. In this paper our main goal is to use the constraints on baseline velocities provided by the GLB868

'Department of Physics, University of Toronto

${ }^{2}$ Harvard-Smithsonian Center for Astrophysics

Copyright 1993 by the American Geophysical Union.

Paper number 93GL02043

0094-8534/93/93GL-02043\$03.00 solution to test the acceptability of a set of published models for the combination of ice history and viscosity profile associated with the North American region.

\section{Method and Results}

In the present analysis we focus on the rates of change of ba line lengths (hereafter "length rates") for sites in Nirth America. We limit our attention to baselines which extend, at least in part, over the near field and periphery of the ancient Laurentide ice sheet. We will not use baselines which include sites on the west coasts of Canada and the contiguous United States, in order to avoid areas of well-known tectonic deformations, nor baselines which include the Ft. Davis VLBI site, which are characterized by anomalous, and as yet unexplained, variations in length. Applying these criteria to the sites included in the GLB868 solution yields a set of 19 baselines; these are listed in Table 1, (together with the length rates obtained in the GLB868 solution) and illustrated in Figure 1. (Ryan et al. [1993] scaled the unc "rtainties so that the reduced $\chi^{2}$ for the series of baselinc estimates is unity. For Richmond-Westford we have usud two significant digits for the $\sigma$ because rounding crrors would be large with respect to the value.)

Our predictions of baseline length rates will be based on the spectral formalism for computing three dimensional motions detailed in Mitrovica et al. [1993b], which is appropriate for the surface loading of a spherically symmetric, self-gravitating, linear visco-elastic Earth model. We adopt a spherical harmonic truncation level at degree and order 256 for the surface mass load. The load will be comprised of a model for the late Pleistocene ice sheets, and a gravitationally self-consistent ocean loading component. The latter is computed using the pseudo-spectral method of Mitrovica and Peltier [19011. The Earth models we consider all have an elastic structure given by the Earth model PREM and a Maxwell visco-elastic rheology. The set of radial viscosity profiles used in the calculations are specified below. In general these profiles are defined by the thickness of an elastic lithosphere (denoted IT), and by a constant value for each of the viscosities in the upper and lower mantle, respectively, $\nu_{U M}$ and $\nu_{L M}$. (The boundary between the upper and lower mantle region is taken to be at $670 \mathrm{~km}$ depth.) Our results will often be summarized in terms of the $\chi^{2}$ residual per degree-of-freedom (here-

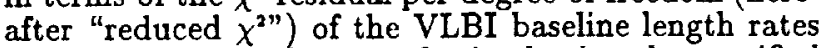
relative to the predictions obtained using the specified combination of ice history and radial viscosity profile. The reduced $\chi^{2}$ computed assuming that glacial isostatic adjustment does not influence the baseline length rates listed in Table 1 (hereafter "the reduced $\chi^{2}$ of the null hypothesis") is 3.3 .

In the following discussion we refer to a "standard" Earth model which has LT $=120 \mathrm{~km}, \nu_{U M}=10^{21} \mathrm{~Pa}$ s and $\nu_{L M}=2 \times 10^{21} \mathrm{~Pa}$ s. In Figure 2 (solid line) we summarize predictions of the length rate of the RichmondWestford baseline for a suite of Earth models identical to the standard model with the exception that $\nu_{L M}$ is varied from $5 \times 10^{20} \mathrm{~Pa}$ s to $5 \times 10^{21} \mathrm{~Pa}$ s. The ice loading used in: the calculation was based on the recently published ICE-3G deglaciation chronology, which extends 
Appendix B. Mitrovica et al. [1993]

$\therefore-4=2, \quad ; \quad+8 \%$ 
TABLE 1. GLB868 Baseline Length Rates

\begin{tabular}{lc}
\hline \multicolumn{1}{c}{ Baseline } & Rate (mm/yr) \\
\hline Aigonquin Park (A)-Gilmore Ck (G) & $3.3 \pm 0.8$ \\
Algonquin Park (A)-Westford (W) & $-0.7 \pm 0.5$ \\
Gilmore Ck (G)-Haystack (H) & $-2.3 \pm 2.2$ \\
Gilmore Ck (G)-NRAO 85 (N1) & $-0.5 \pm 1.4$ \\
Gilmore Ck (G)-Platteville (P) & $5.6 \pm 2.3$ \\
Gilmore Ck (G)-Richmond (R) & $1.1 \pm 1.2$ \\
Gilmore Ck (G)-Westford (W) & $-0.4 \pm 0.5$ \\
Gilmore Ck (G)-Whitehorse (Wh) & $-3.8 \pm 3.8$ \\
Beltsville (B)-NRAO 85 (N1) & $3.4 \pm 1.3$ \\
Britsville (B)-Richmond (R) & $6.9 \pm 2.5$ \\
Beltsville (B)-Westford (W) & $0.0 \pm 3.0$ \\
IIaystack (H)-NRAO 140 (N2) & $1.4 \pm 2.0$ \\
Maryland Pt (M)-Richmond (R) & $0.6 \pm 4.4$ \\
Miryland Pt (M)-Westford (W) & $1.4 \pm 1.5$ \\
NRAO 85 (N1)-Riclimond (R) & $2.2 \pm 0.9$ \\
NRAO 85 (N1)-Westford (W) & $4.0 \pm 2.0$ \\
NRAO 140 (N2)-Westford (W) & $0.5 \pm 0.3$ \\
Platteville (P)-Westford (W) & $3.3 \pm 2.2$ \\
Richmond (R)-Westford (W) & $-0.2 \pm 0.15$ \\
\hline
\end{tabular}

from 18 to $5 \mathrm{kyr}$ before present (Tushingham and Peltier [1991]), and adapted in order to incorporate a growth phase for the late Pleistocene ice sheets (see Mitrovica and Peltier [1993]).

The analysis of Tushingham [1991] represents the only at tempt to date to calculate baseline length rates due to glacial isostatic adjustment. However, he assumed that these rates could be accurately computed by considering only radial deformations at the terminating sites. To assess the validity of this assumption, we also show in Figure 2 (dashed line) the component of the baseline Icng,th rate which is due to radial motions at the Rich-

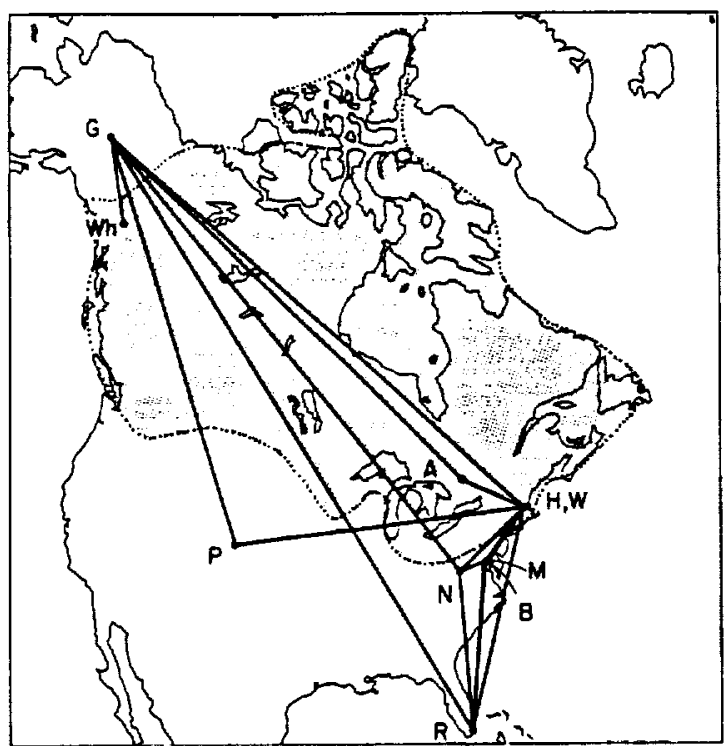

Fig. 1. The VLBI baselines included in this study. The letters on the figure denote specific VLBI sites, as indicated in Table 1. The shaded region on the plot shows the geographic extent of the North American ice cover during the last glacial maximum according to the ICE3G morlel of Tushingham and Peltier [1991].

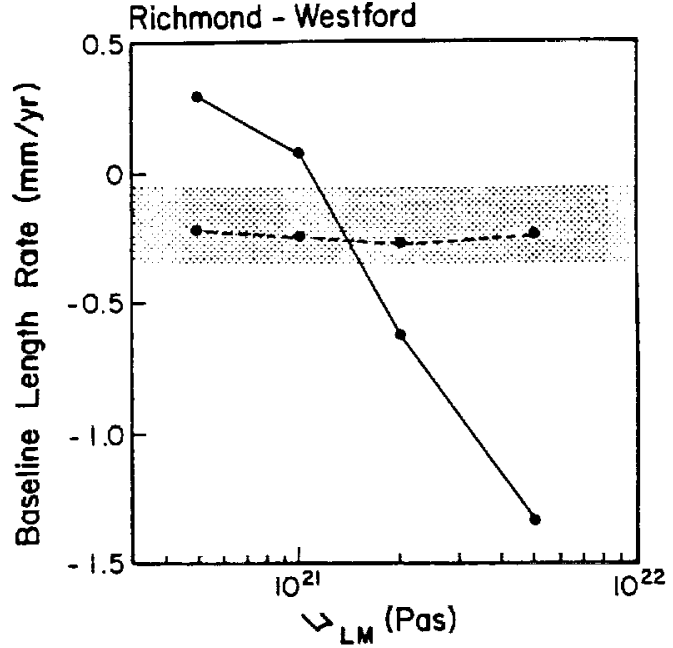

Fig. 2. The predicted length rate of the RichmondWestford baseline as a function of $\nu_{L M}$. The solid line was computed from the three-dimensional motion of the Richmond and Westford sites, while the dashed line was generated assuming that only radial motions at these sites contribute to the length rate. The shaded region represents the VLBI determined constraint $( \pm 1 \sigma)$ on the baseline length rate (Table 1 ).

mond and Westford sites. It is clear that the Tushinghain [1991] assumption is, in general, invalid. Indeed, over the range of Earth models considered in Figure 2 the horizontal motions of the end sites generally contribute more to the baseline length rate than do the associated radial motions. Clearly, inferences of viscosity based upon predictions generated using the Tushingham [1991] approximation would be suspect.

In Figure 3 we plot the reduced $\chi^{2}$ for the comparison of the VLBI baseline length rates with the predictions generated from many Earth models and two ice models. Figure 3a provides results generated using the ice loading adapted from the ICE-3G deglaciation chronology; each curve connects results from a set of Earth inodcls constructed by varying a specific characteristic of the standard model (see caption). The two curves in Figur. $3 \mathrm{~b}$ are distinguished by the ice loading history adopted in the calculation. In particular, we generated the points on the dashed-dotted line using the ICE-1 model of Peltier and Andrews [1976] (adapted in the manner described above).

The results in Figure 3 show that the Earth models and icc histories represented there do not yield predictions with reduced $\chi^{2}$ values significantly less than the value for the null hypothesis (3.3). The reasons for this outcome may be some combination of errors in the details of the ice loading histories over North America, the limited class of Earth models considered (see below), and contributions from neglected geophysical proccsses (e.g., tectonic deformations). Nevertheless, two important conclusions may be made. First, some ice history/Earth model parings predict length rates which appear to be incompatible with the VLBI determined constraints, and may as a consequence be ruled out. For example, variants of the standard Earth model with LT $\leq 80$ or $\nu_{L M} \geq 3.3 \times 10^{21}$ Pas, together with the ICE-3G deglaciation chronology, yield reduced $x^{2}$ residuals in cxcess of 6.6 (or larger than twice the value for the null hypothesis). In contrast, many Earth models do not produce cxtremely high reduced $\chi^{2}$ values, and therefore cinnot be rejected. 


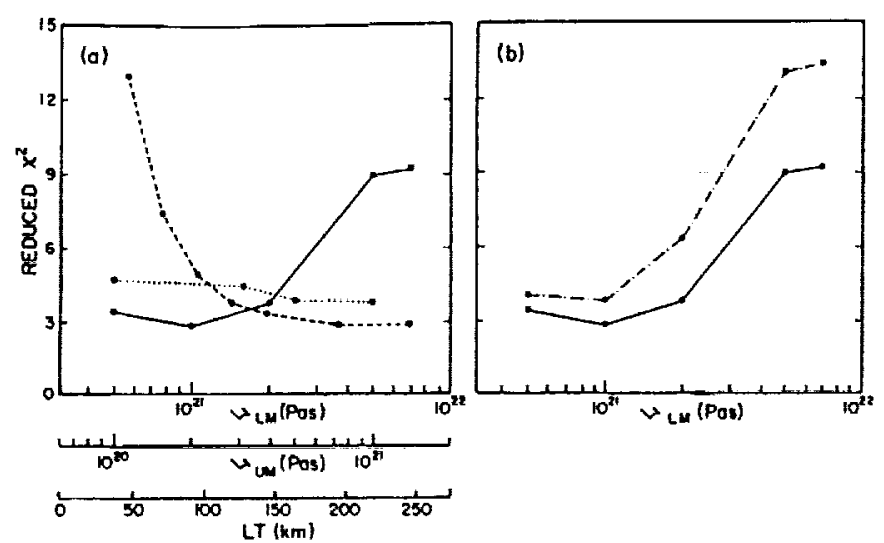

Fig. 3. The reduced $\chi^{2}$ residual for the VLBI determined baseline length rates in Table 1 relative to the corresponding predictions obtained from a suite of Earth models and ice histories. (a) All curves are computed using the ice loading history adapted from the ICE-3G deglaciation chronology. The solid, dotted, and dashed lines are generated by varying, respectively, $\nu_{L M}, \nu_{U_{M}}$, and LT of the standard Earth model. Each curve has an associated abscissa scale. (b) Both curves are generated by varying $\nu_{L M}$ of the standard Earth model. The solid and dashed-dotted lines were computed using ice loading histories adapted from, respectively, the ICE-3G and ICE-1 deglaciation models.

The predicted length rates for a subset of six of the 19 baselines, for each family of results shown in Figure 3a, are given in Figure 4. The dramatic increase in the reduced $\chi^{2}$, for Earth models with progressively thinner lithospheres, is clearly the consequence of a misfit between predictions and observations for almost all the baselines considered in Figure 4, suggesting a rather systematic preference for Earth models with relatively thick lithospheres (i.e., LT $\geq 100 \mathrm{~km}$ ). The pronounced increase in the reduced $\chi^{2}$, for $\nu_{L M}$ greater or less than a value near $10^{21} \mathrm{I}$ 'a $\mathrm{s}$, is due in large part to misfits in the predictions associated with the Richmond-Westford briscline. As an example, increasing $\nu_{L M}$ from $2 \times 10^{2:} \mathrm{Pa}$ $s$ to $5 \times 10^{23}$ Pas results in an increase of the reduced $\chi^{2}$ by apprisximately 5.2 in Figure 3a. The increased misfit for $\mathrm{I}$ (c)lictions based on these two lower mantle viscositics, for the Richmond-Westford baseline length rate alone, accounts for $50 \%$ of this increase. (Mitrovica et al. [1993a] have found that far field sites, such as Richmond, have motions which are drawn more strongly toward the previously glaciated region (in this case Laurentia) as $\nu_{L M}$ is increased. This trend gives rise to a transition from a lengthening to a shortening of the Richmond-Westford baseline as $\nu_{L M}$ is increased.)

From Figure 3 we conclude that the current VLBI data set of North American baseline length rates may be applied to assess the acceptability of specific pairings of ic and Earth models. Inferences of the ice cover over North $A$ merica, and the rheology below the region, have been a source of active interest and contention in the geophysical literature (see below). In the following we consider a number of ice model/Earth model combinations which have been proposed for the North American region on the basis of a variety of independent geophysical and geological constraints. The defining characteristics of four particular models are given in Table 2.

The column labelled T\&P in Table 2 summarizes the model of Tushingham and Peltier [1991, 1992] which combines the standard Earth model and the ICE-3G

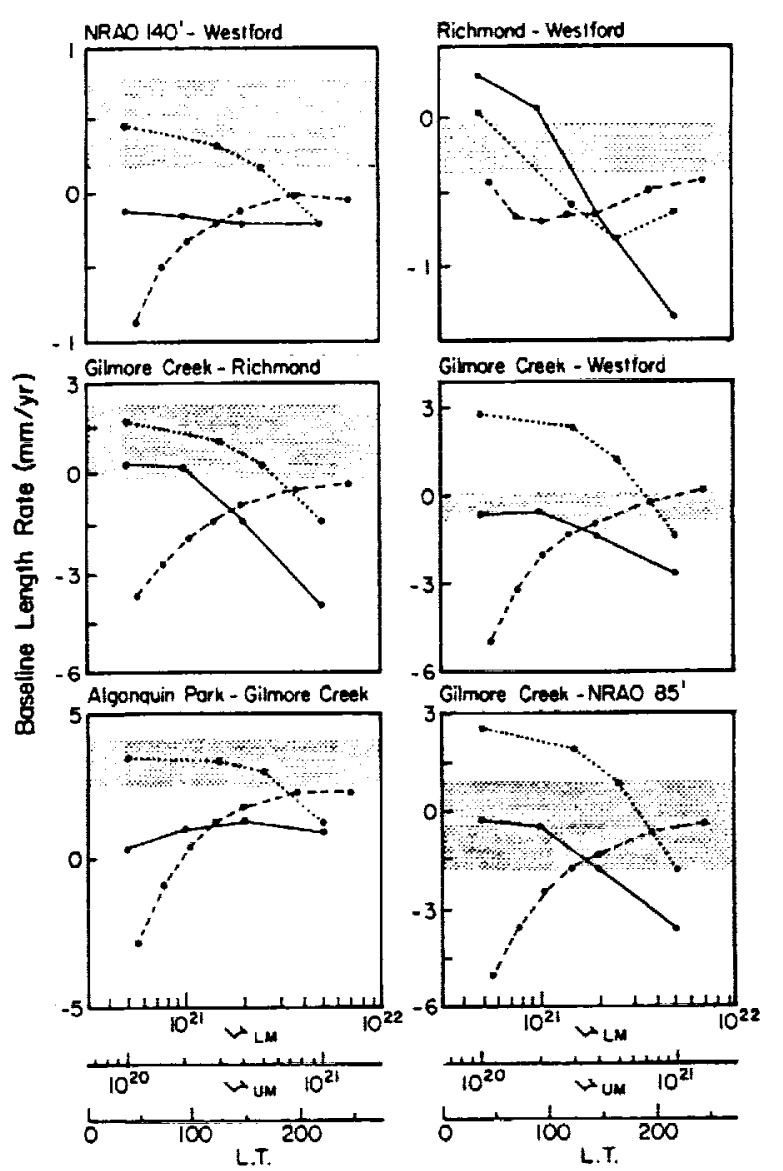

Fig. 4. The predicted baseline length rates for six baselines (as labelled) computed using the ice loading history adapted from the ICE-3G deglaciation chronology. The solid, dotted and dashed lines are generated by varying, respectively, $\nu_{L M}, \nu_{U_{M}}$ and LT of the standard model. The shaded region on each frame represents the VLBI determined constraint $( \pm 1 \sigma)$ on the baseline length rate.

deglaciation chronology; they have argued that this combination satisfies a globally distributed data base of RSL curves, including those collected in North America. The second model is that of Cathles [1975] (CAT) and includes an ice history that is similar to the ICE-1 model of Peltier and Andrews [1976]. The CAT model

TABIE 2. Summary of Models ${ }^{\dagger}$ and Results

\begin{tabular}{lccccc}
\hline Parameter & \multicolumn{5}{c}{ Model Values } \\
& T\&P & CAT & N\&L & DMVC & Null \\
\hline$\nu_{U M}^{\dagger \dagger}$ & 1.0 & 1.0 & 0.5 & 1.0 & \\
$\nu_{L M}^{\dagger \dagger}$ & 2.0 & 0.9 & 30.0 & $1.0-10.0$ \\
LT $(\mathrm{km})$ & 120.0 & 70.0 & 100.0 & 120.0 \\
\hline Baseline & \multicolumn{5}{c}{ Reduced $\chi^{2}$} \\
\hline N2-W & 0.3 & 1.7 & 0.1 & 0.1 & 0.1 \\
R-W & 0.4 & 0.0 & 8.4 & 0.1 & 0.1 \\
G-R & 0.2 & 0.1 & 0.1 & 0.1 & 0.0 \\
G-W & 0.2 & 0.5 & 1.7 & 0.0 & 0.0 \\
A-G & 0.4 & 3.3 & 0.1 & 0.2 & 0.9 \\
G-N1 & 0.1 & 0.1 & 0.0 & 0.0 & 0.0 \\
All 19 & 3.8 & 7.9 & 13.7 & 2.4 & 3.3 \\
\hline
\end{tabular}

${ }^{\dagger}$ Ice models are discussed in text.

${ }^{\dagger \dagger} \nu_{U M}$ and $\nu_{L M}$ are in units of $10^{21} \mathrm{~Pa}$. 
was meant to satisfy the constraints imposed by the sea level record in North America, and the decay time inferred from that record. In contrast to these inferences, Nakada and Lambeck [1991] (N\&L) have argued for a large (factor of 60) viscosity jump between the upper and lower mantle. The N\&L model was based on fits to sea level curves in the Hudson Bay region, and their global ice model (ARC3 + ANT3) adopts the ICE-1 chronology over North America. The final model, labelled DMVC (Deep Mantle Viscosity Contrast), denotes a model characterized by an order of magnitude increase in viscosity at $1900 \mathrm{~km}$ depth in the mantle. Above $1900 \mathrm{~km}$ depth the viscosity is $10^{21} \mathrm{~Pa} \mathrm{~s}$. This viscosity model was proposed by Mitrovica and Peltier [1992] as one which might reconcile the sea level record over Hudson Bay, and the independent inferences of viscosity provided by data associated with mantle convection (see Mitrovica and Peltier, 1992, for details). The Mitrovica and Peltier [1992] calculations adopted the ICE-3G deglaciation history.

The remaining rows of Table 2 provide the total reduced $\chi^{2}$ for the VLBI determined length rates in Table 1 relative to predictions based on the models described above (see row "All 19"), as well as the contribution to this reduced $\chi^{2}$ from predictions for each of the six baselines considered in Figure 4 . The lowest (total) reduced $\chi^{2}$ values are obtained for the models DMVC and T\&P. These reduced $\chi^{2}$ values are, however, indistinguishable, at the $95 \%$ confidence limit, from each other and from the value for the null hypothesis.

As a consequence of its low reduced $\chi^{2}$ the combination of ice history and Earth rheology proposed by Tushingham and Peltier $[1991,1992]$ cannot be ruled out $b y$ the VLBI constraints. The pairing proposed by Cathles [1975] yields a reduced $x^{2}$ about double the $T \& P$ value; the results in Table 2 suggest that the CAT model performs particularly poorly in regard to predictions of length rates for the NRAO $140^{\prime}$ - Westford and Algonquin Park - Gilmore Creek baselines. Figures 3 and 4 indicate that the reduced $x^{2}$ residual might be lowered if the thickness of the lithosphere in the Cathles [1975] model was increased. Indeed, an increase of LT to $120 \mathrm{~km}$ in the CAT model has been found to lower the reduced $\chi^{2}$ value below 4 .

The total reduced $\chi^{2}$ computed for the N\&L ice history/Earth rheology is relatively much higher. The predicted length rate of the Richmond - Westford baseline is a primary contributor to the misfit associated with this model. Indeed, the Nakada and Lambeck [1991] model predicts a shortening of approximately $2 \mathrm{~mm} / \mathrm{yr}$ for the Richmond - Westford baseline length rate, which differs significantly from the observational constraint. The location of Richmond, in the far field of the Laurentide ice sheet, suggests that the viscosity model proposed by these authors would almost certainly have to be altered in order to satisfy the VLBI determined constraint on the Richmond - Westford baseline.

The reduced $\chi^{2}$ associated with the model DMVC is about $25 \%$ lower than the value for the null hypothe,is. The result indicates that a relatively large viscosity contrast with depth within the lower mantle is not ruled out by the VLBI determined constraints on the baseline length rates listed in Table 1 . Furthermore, it indicates that the sensitivity of the reduced $\chi^{2}$, to variations in $\nu_{L M}$ (Figure 3 ), is more accurately identified as a sensitivity to variations in viscosity above $1900 \mathrm{~km}$ depth in the lower mantle (see also Mitrovica et al., 1993a).
Acknowledgements. This work was supported by an NSERC of Canada Research Grant, NASA grant NAG51930, and by the Smithsonian Institution.

\section{References}

Cathls, L. M., The Viscosity of the Earth's Mantle, Princeton University Press, Princeton, N.J., 1975.

James, T. S., and W. J. Morgan, Horizontal motions due to postglacial rebound, Geophys. Res. Lett., 17, 957-960, 1990.

Mitrovica, J.X., and W.R. Peltier, On post-glacial geoid relaxation over the equatorial oceans, J. Geophys. Res., 96, 20053-20071, 1991.

Mitrovica, J.X., and W.R. Peltier, Constraints on mantle viscosity from relative sea level variations in Hudson Bay, Geophys. Res. Lett., 19, 1185-1188, 1992.

Mitrovica, J.X., and W.R. Peltier, Present day secular variations in the zonal harmonics of the Earth's geopotential, J. Geophys. Res., 98, 4509-4526, 1993.

Mitrovica, J.X., J. L. Davis, and I. I. Shapiro, A spectral formalism for computing three-dimensional deformations due to surface loads, 2. Glacial Isostatic Adjustment, J. Geophys. Res., submitted, 1993a.

Mitrovica, J.X., J. L. Davis, and I. I. Shapiro, A spectral formalism for computing three-dimensional deformations due to surface loads, 1. Theory, J. Geophys. Res., submitted, 1993b.

Nakada, M. and K. Lambeck, Late Pleistocene and Holocene sea-level change: Evidence for lateral mantle viscosity structure?, in Glacial Isostasy, Sea-Level and mantle Rheology, pp. 79-94, proceedings of the NATO Advanced Research Workshop on Glacial Isostasy, Sea-Level and Mantle Rheology, ed. Sabadini, R., K. Lambeck, and E. Boschi, NATO ASI Series v. 334, Kluwer, The Netherlands, 1991.

Peltier, W. R., and J. T. Andrews, Glacial isostatic adjustment - I. The forward problem, Geophys. J. R. Astron. Soc., 46, 605-646, 1976.

Ryan, J. W., C. Ma, and D. S. Caprette, NASA Space Geodesy Program - GSFC Data Analysis-1992, NASA Tech. Mem. 104572, NASA GSFC, Greenbelt, Maryland, 471 pp., 1993.

Tushingham, A. M., Potential effects of ongoing postglacial adjustment on very long baseline interferometry measurements, Geophys. Res. Lett., 18, 1281$1284,1991$.

Tushingham, A. M, and W. R. Peltier, ICE-3G: A new global model of late Pleistocene deglaciation based upon geophysical predictions of postglacial relative sea level change, J. Geophys. Res., 96, 4497, 1991.

Tushingham, A. M. and W. R. Peltier, Validation of the ICE-3G model of Wurm-Wisconsin deglaciation using a global data base of relative sea level histories, J. Geophys. Res., 97, 3285-3304, 1992.

J. X. Mitrovica, Department of Physics, University of Toronto, Toronto, Ontario, Canada M5S 1 A7.

J. I. Davis \& I. I. Shapiro, Harvard-Smithsonian Center for Astrophysics, 60 Garden St., Cambridge, MA.

(Received April 29, 1993;

Accepted July 22, 1993.) 
